\title{
NBS
}

Eechnical Note

$\eta_{0.303}$

\section{EXTENSION OF PROGRAMS FOR CALCULATIONS OF GREAT CIRCLE PATHS AND SUNRISE-SUNSET TIMES}

J. H. Crary

U. S. DEPARTMENT OF COMMERCE NATIONAL BUREAU OF STANDARDS 


\section{THE NATIONAL BUREAU OF STANDARDS}

The National Bureau of Standards is a principal focal point in the Federal Government for assuring maximum application of the physical and engineering sciences to the advancement of technology in industry and commerce. Its responsibilities include development and maintenance of the national standards of measurement, and the provisions of means for making measurements consistent with those standards; determination of physical constants and properties of materials; development of methods for testing materials, mechanisms, and structures, and making such tests as may be necessary, particularly for government agencies; cooperation in the establishment of standard practices for incorporation in codes and specifications; advisory service to government agencies on scientific and technical problems; invention and development of devices to serve special needs of the Government; assistance to industry, business, and consumers in the development and acceptance of commercial standards and simplified trade practice recommendations; administration of programs in cooperation with United States business groups and standards organizations for the development of international standards of practice; and maintenance of a clearinghouse for the collection and dissemination of scientific, technical, and engineering information. The scope of the Bureau's activities is suggested in the following listing of its four Institutes and their organizational units.

Institute for Basic Standards. Electricity. Metrology. Heat. Radiation Physics. Mechanics. Applied Mathematics. Atomic Physics. Physical Chemistry. Laboratory Astrophysics." Radio Standards Laboratory: Radio Standards Physics; Radio Standards Engineering.** Office of Standard Reference Data.

Institute for Materials Research. Analytical Chemistry. Polymers. Metallurgy. Inorganic Materials. Reactor Radiations. Cryogenics. * Office of Standard Reference Materials.

Central Radio Propagation Laboratory.* Ionosphere Research and Propagation. Troposphere and Space Telecommunications. Radio Systems. Upper Atmosphere and Space Physics.

Institute for Applied Technology. Textiles and Apparel Technology Center. Building Research. Industrial Equipment. Information Technology. Performance Test Development. Instrumentation. Transport Systems. Office of Technical Services. Office of Weights and Measures. Office of Engineer. ing Standards. Office of Industrial Services.

* NBS Group, Joint Institute for Laboratory Astrophysics at the University of Colorado.

** Located at Boulder, Colorado. 


\title{
NATIONAL BUREAU OF STANDARDS Eechnical Note 303 \\ Issued February 18, 1965
}

\section{EXTENSION OF PROGRAMS FOR CALCULATIONS \\ OF GREAT CIRCLE PATHS AND SUNRISE-SUNSET TIMES}

\author{
J. H. Crary \\ Central Radio Propagation Laboratory \\ National Bureau of Standards \\ Boulder, Colorado
}

NBS Technical Notes are designed to supplement the Bureau's regular publications program. They provide a means for making available scientific data that are of transient or limited interest. Technical Notes may be listed or referred to in the open literature.

For sale by the Superintendent of Documents, U.S. Government Printing Office Washington D.C. 20402

Price: 25 cents 

1. Introduction .................... . . I

2. The Computer Programs . . . . . . . . . . . . 2

2.1 Introduction ................ 2

2.2 Sunrise-Sunset Program (DALITP) . . . . . . . . 3

2.3 Sunrise-Sunset Program with Bearing Calculations (DALITB)

2.4 Great Circle Path Subroutine (PATH) . . . . . . . 4

2.5 Solar Angle Subroutine (SOLANG) . . . . . . . . 9

3. Some IlIustrations of the Results for Iong and Short Paths II

4. Acknowledgments ................. . . 13

5. References . . . . . . . . . . . . . . 13

Figures . . . . . . . . . . . . . . . . . . . 14 



\section{EXTENSION OF PROGRAMS FOR CALCULATIONS OF GREAT CIRCLE PATHS AND SUNRISE-SUNSET TTMES}

\section{J. H. Crary}

A previous note by Brady and Crombie [1964] describes methods and a program for calculating and plotting sunrise and sunset times at specified heights and distances along a great circle path determined by the locations of the end points. The program also plots the distribution of darkness or daylight along the path versus time.

This program has been modified to remove some restrictions on the use of the earlier program, and to provide the option of calculating the short or long great circle path. In addition, transmitter, receiver, and solar bearings are also calculated and plotted. Some interesting characteristics of the short and long paths from Rugby, England, to Byrd Station, Antarctica, are shown.

\section{Introduction}

A previous note by Brady and Crombie [1964] described the methods and gave the formulas for the calculation of sunrise and sunset times at specified heights and distances along a great circle path. The program previously described had some limitations which restricted its use. Modifications were made to the program to remove the preceding restrictions and to add some additional features. 
These features are the calculation of (1) either the short or long path, (2) the transmitter bearing at each segment of the path, (3) the solar bearing angle at sunrise and sunset at each segment, (4) the difference angle between the transmitter and solar bearings, and (5) the cosine of this difference angle. A routine was then added to plot these results, together with the percentage of darkness along the path. The necessity of giving the easternmost end of the path first was also removed, since the choice depends upon whether the short or long path is desired.

These modifications were accomplished by adding three subroutines to the original program. These programs and subroutines are available in Fortran language. A description of these will be given which includes the necessary equations and logical statements to enable a person to calculate these results manually or by means of a computer program, without first determining how to make the necessary decisions; this is a time consuming process and need not be duplicated.

\section{The Computer Programs}

\subsection{Introduction}

The computer programs consist of two main Fortran programs, DALITP and DALITB, together with the necessary subroutines (PATH, SOIANG, DATBX, PLOT). The functions of these subroutines are: (1) PATH--to calculate the necessary information about the great circle path, such as the total length, the bearing angles of the transmitter and receiver, and the position of points at specified intervals along the path; (2) SOLANG--to 
calculate the solar zenith and bearing angles; (3) DATBX--a function which furnishes the date on which the calculations are performed to the calling program; and (4) PLOT--a general output plotting routine. The functions of these subroutines are discussed in 2.2. - 2.5. The following notation will be used in the description:

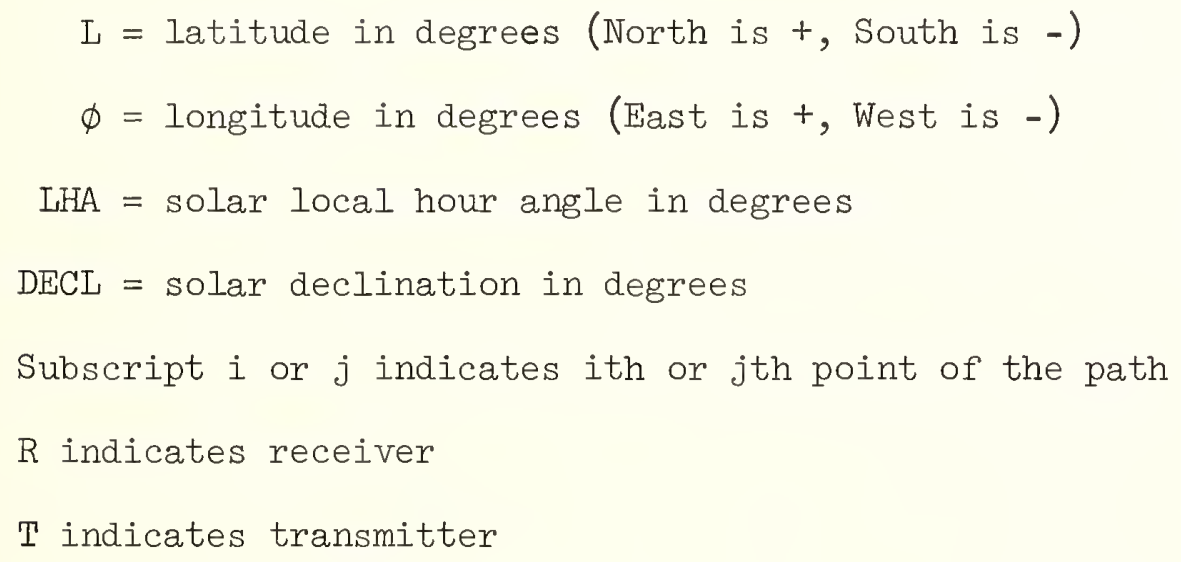

\subsection{Sunrise-Sunset Program (DALITP)}

This program is the modified version of the original DALIT program and controls the data card reading and the output printing. It also performs the calculations of the sunrise and sunset times, using the same equations given by Brady and Crombie [1964].

In the case of $|\cos L H A|>1$, the following test is made:

$$
\begin{aligned}
& \text { if }\left(I_{i} \cdot D E C L\right)<0 \text { the path is always dark, } \\
& \text { if }\left(I_{i} \cdot D E C L\right) \geq 0 \text { the path is always light. }
\end{aligned}
$$

This takes care of both northern and southern hemisphere conditions. The data inputs are the same as before except for the following 
changes: (1) a given number of pairs of ionosphere and screen heights, up to a maximum of ten, may be used, and (2) the option of calculating the long or short path is provided.

\subsection{Sunrise-Sunset Program with Bearing Calculations (DALITB)}

This program is the same as DALITP, with the addition of the subroutine SOLANG to calculate the solar bearing angles between the solar and transmitter bearing, and the cosine of this difference. The PLOT subroutine then is used to plot the solar bearings, the cosine of the difference angle, and the distance along the path where the terminator* crosses, as a function of time.

\subsection{Great Circle Path Subroutine (PATH)}

This subroutine is called by the main program to do the great circle calculations. The program has several options to calculate for the short or long path: (1) distance between two specific points; (2) latitude, longitude, and bearing of transmitter at given intervals on a given path; (3) coordinates of reflection points for an n-reflection ray between given points; or (4) coordinates of the end points of a path when given one end and the jth point on an n-reflection path.

The option of being able to calculate either the short or the long great circle path between two specified points requires several logical

\footnotetext{
* Terminator--a useful astronomical term for the line separating the dark and sunlit portions of a planet, applied here at the ionospheric height specified.
} 
decisions to be made at different points during the calculation in order to insure that the proper solution is being obtained. The spherical triangles involved in these solutions are illustrated in figure l. The polar angle of the spherical triangle is the difference in longitude between the two points on the path being considered. The algebraic sign of the difference is not used in the calculations. Four cases can actually be defined, depending upon whether the short or long path is desired, and whether the transmitter or starting end of the path is the eastern-most or western-most point of the path. This decision depends upon whether the short or long path is desired.

In addition, decisions must be made as to which of the possible solutions to equations involving $\cos ^{-1}$ will yield the desired path. It is also necessary to account for some of the cases of the degenerate spherical triangle, such as where both points of the path lie on a meridian, etc. For this reason, the necessary equations and logic statements are included, permitting a straightforward calculation of the desired path. The flagl mentioned in the following description is a logical variable which indicates a decision made on the basis of the orientation of transmitter and receiver and whether the short or long path is desired.

Calculate the polar angle or longitude difference, $\left|\phi_{\mathrm{T}}-\phi_{\mathrm{R}}\right|$ (see figure 1).

(2.1) If $\left|\phi_{T}-\phi_{R}\right| \leq 180^{\circ}$, continue; if $>180^{\circ}$, go to (2.2).

(2.1.1) If short path, $\phi=360-\left|\phi_{T}-\phi_{R}\right|$ and set flagl = 2, go to (3). 
(2.1.2) If long path, $\phi=\left|\phi_{\mathrm{T}}-\phi_{\mathrm{R}}\right|$, and set flagl $=1$, go to (3). (2.2) If $\left|\phi_{\mathrm{T}}-\phi_{\mathrm{R}}\right|>180^{\circ}$.

(2.2.1) If short path, $\phi=\left|\phi_{T}-\phi_{R}\right|$, and set flagl = I, go to (3). (2.2.2) If long path, $\phi=360-\left|\phi_{T}-\phi_{R}\right|$, and set flagl $=2$.

Calculate $D$, the total path distance.

(3.1) If $\cos I_{R} \neq 0$, go to (3.2); if $=0$, continue. If $\cos I_{R}=0$, then $\operatorname{DRAD}=\left|\mathrm{I}_{\mathrm{T}}-\mathrm{I}_{\mathrm{R}}\right| \cdot \pi / 180$ radians.

(3.1.1) If $I_{R}=0$, impossible branch, error message, exit.

(3.1.2) If $I_{R}<0, T=\pi$ rad, where $T$ is the angle of the spherical path triangle at the transmitter.

(3.1.3) If $I_{R}>0, T=0$.

(3.1.4) If long path $D R A D=D R A D-2 \pi . D=D R A D \cdot R$ where $R$ is the effective earth radius. $R$ is taken as the temperate latitude average of the radii determined from the polar and equatorial circumferences, or $\left(3 R_{e q}+R_{p o l}\right) / 4$. The calculation then proceeds to (4).

(3.2) If $\cos I_{R} \neq 0, D R A D=\cos ^{-1}\left[\sin I_{T} \sin I_{R}+\cos I_{T} \cos I_{R}\right.$ $\cos \phi]$ from the cosine law for the spherical triangle shown in figure 1.

(3.2.1) If $\sin (D R A D)=0$, then end points are antipodal, and there are an infinite number of solutions. This causes a message to be printed and the calculation is terminated.

(3.2.2) If $\sin (D R A D) \neq 0$ : for long path $D R A D=2 \pi-D R A D$; for both paths $D=D R A D \cdot R$. If only distance is desired, return to main program. 
(3.2.3) Determine number of segments. Complete calculation of angle T.

(4.1) If $\cos \mathrm{L}_{\mathrm{T}}=0$, continue; if $\neq 0$, go to (4.3).

(4.I.I) If $\mathrm{I}_{\mathrm{T}}=0$, error message, exit.

(4.1.2) If $\mathrm{I}_{\mathrm{T}}<0, \mathrm{~T}=0$, go to $(4.1 .4)$.

(4.1.3) If $I_{T}>0, T=\pi$.

(4.1.4) Then $I_{j}=I_{T}-\left(I_{T} /\left|I_{T T}\right|\right) D_{j} / R, B_{j}=90-\left(I_{T} /\left|I_{T}\right|\right) 90, \phi_{j}=$ $\phi_{R} ;$ go to $(4 \cdot 3)$.

(4.2) If $\cos I_{T} \neq 0$.

(4.3) $T=\cos ^{-1}\left[\left(\sin L_{R}-\cos D \sin L_{T}\right) /\left(\cos I_{T} \sin D\right)\right]$ from the law of cosines again applied to the path triangle of figure $l$.

(5) Calculate the latitudes at the jth segment along the frth: $L_{j}=90^{\circ}-\cos ^{-1}\left(\sin L_{T} \cos D_{j}+\cos L_{T} \sin D_{j} \cos T\right)$. This is another variation of the law of cosines applied to the triangle of the pole, point $j$ and the transmitter. The use of $90-\cos ^{-1}$ instead of $\sin ^{-1}$ removes an ambiguity in the result.

(5.1) If $\cos \mathrm{L}_{j}=0, \Delta \phi=0$, since point $j$ is at a pole. Here $\Delta \phi$ is the difference in longitude between the transmitter and point $j$. Go to $(6.3)$.

(5.2) If $\cos \mathrm{L}_{j} \neq 0$.

(6) Calculate the longitudes.

(6.1) $\operatorname{Cos} \Delta \phi=\left(\cos D_{j}-\sin L_{T} \sin L_{j}\right) /\left(\cos L_{T} \cos L_{j}\right)$. This also comes from the law of cosines. 
(6.2) Now, using the law of sines for $\sin \Delta \phi: \sin \Delta \phi=(\sin \mathrm{T} \sin$ $\left.D_{j}\right) /\left(\cos L_{j}\right)$.

(6.2.1) If $\sin \Delta \phi \geq 0, \Delta \phi=\cos ^{-1}(\cos \Delta \phi)$, go to (6.3).

(6.2.2) If $\sin \Delta \phi<0, \Delta \phi=360-\cos ^{-1}(\cos \Delta \phi)$.

(6.3) If $\left(I_{T}-I_{R}\right) \geq 0$, continue; if $<0$, go to $(6.4)$.

(6.3.1) If flagl $=1[\operatorname{see}(2.1)-(2.2)], \phi_{j}=\phi_{T}-\Delta \phi$, go to (7).

(6.3.2) If flagl $=2, \phi_{j}=\phi_{T}+\Delta \phi$, go to (7).

(6.4) If $\left(I_{T}-I_{R}\right)<0$ and

(6.4.1) If flagl $=1$ use (6.3.2), go to (7).

(6.4.2) If flagl $=2$ use $(6 \cdot 3 \cdot 1)$.

(7) Check longitude for proper hemisphere

(7.1) If $\left|\phi_{j}\right|-180 \leq 0$, continue; if $>0$, go to (8).

(7.1.1) If $\phi_{j}<0, \phi_{j}=\phi_{j}+360^{\circ}$, go to (8).

(7.2) If $\left|\phi_{j}\right| \geq 0, \phi_{j}=360^{\circ}+\phi_{j}$.

(8). Find transmitter-bearing $B_{j}$.

(8.1) If $\cos I_{R}=\phi, B_{j}=90+\left(I_{R}\right) /\left(\left|I_{R}\right|\right) \cdot 90$, go to (9).

(8.2) Or if $\cos I_{R} \neq 0, B_{j}=\cos ^{-1}\left[\left(\sin I_{T}-\sin I_{j} \cos D_{j}\right) /\right.$ $\left.\left(\cos \mathrm{L}_{j} \sin \mathrm{D}_{j}\right)\right]$ again from the law of cosines.

(8.3) If $\left(\phi_{T}-\phi_{R}\right)<0$, continue; if $\geq 0$, go to (8.4).

(8.3.1) Flag $I=1[\operatorname{see}(2.1)-(2.2)] \mathrm{B}_{j}=360-\mathrm{B}_{j}$, go to (9).

(8.3.2) Or if flagl $=2, B_{j}=B_{j}$, go to $(9)$.

(8.4) If $\left(\phi_{\mathrm{T}}-\phi_{\mathrm{R}}\right) \geq 0$.

(8.4.1) And flagl $=1$, use (8.3.2), go to (9).

(8.4.2) Or if flag $I=2$, use (8.3.1). 
(9) Find receiver bearing from transmitter.

(9.1) If $\left(\phi_{\text {T }}-\phi_{R}\right)<0$, continue; if $\geqq 0$, go to (9.2).

(9.1.1) And flagl $=1, B_{R}=T$, go to (10).

(9.1.2) Or if flagl $=2, B_{R}=360-T$, go to (10).

(9.2) If $\left(\phi_{\text {TI }}-\phi_{\mathrm{R}}\right) \geq 0$ 。

(9.2.1) And flagl = 1 use (9.1.2), go to (10).

(9.2.2) Or if ilagl $=2$ use $(9.1 .1)$.

(10) Since the transmitter bearing is undefined at the transmitter, we use:

(10.1) $\quad B_{1}=B_{R}+180$.

(10.2) If $\left(B_{1}-360\right) \geq 0, B_{1}=B_{1}-360$, go to (II).

(10.3) Or if $\left(B_{1}-360\right)<0, B_{1}=B_{1}$.

(11) The subroutine returns to the main program here.

\subsection{Solar Angle Subroutine (SOI_ANG)}

This subroutine finds the solar bearing angles $S_{i}$ and the zenith angles $x_{i}$.

The inputs are an array of $\mathbb{N}$ sets of path latitude (XIAT) and longitude (XION), solar declination (DECL), and Greenwich hour angle (GHA), where $\mathbb{N} \leqq 1000$. The variable NTTMES may be used to specify whether there is only one pair of values (NTIMES $=1$ ) for GHA and DECL, or whether there is a pair for each point (NTTMES > 1).

The logic statements given here are used to find the proper solution to equations involving the arc cosine function. It is necessary to pick 
the solution corresponding to the desired triangle. It is also necessary to make decisions so that the solar bearing angle will follow the convention of being measured clockwise from North and the solar zenith angle will lie between $-180^{\circ}$ and $+180^{\circ}$.

The calculation then proceeds as follows, using the solar triangle of figure 2.

$$
(1.3 .1)
$$

Find the solar local hour angle SLHA $=$ GHA $+\phi_{i}$. If SLHA $<0$, then SLHA $=$ SLHA +360 , go to $(1.3)$. If SLHA $\geq 0$ then If $($ SLHA - 360) $\geq 0$, continue; if < 0 , go to (1.4).

(1.3.1) SLHA = SLHA - 360, go to (2). If $($ SLHA -360$)<0$, SLHA = SLHA.

Calculate $x_{i}=\cos ^{-1}\left(\sin I_{i} \sin \mathrm{DECL}+\cos \mathrm{L}_{i} \cos \mathrm{DECL}\right.$ cos SLHA) from the law of cosines. Next find the bearing angle.

(2.I) If $\left|I_{i}\right|-90=0$, continue; if $\neq 0$, gc to (2.2).

(2.1.1) $S_{i}=$ SLHA, go to $(2.5)$.

(2.2) If $\left|I_{i}\right|-90 \neq 0$.

(2.3) If $(x|x|-180)=0$, continue; if $\neq 0$, go to (2.4).

(2.3.1) And if $I_{i}-D E C I<0, S_{i}=180^{\circ}$, go to (2.5).

(2.3.2) Or if $I_{i}-D E C L \geq 0, S_{i}=0^{\circ}$, go to (2.5).

(2.4) If $x(|x|-180) \neq 0$.

(2.4.1) $S_{i}=\cos ^{-1}\left[\left(\sin D E C L-\sin L_{i} \cos x\right) /\left(\cos L_{i} \sin x\right)\right]$ from the law of cosines.

(2.5) If $($ SLHA $-I 80)<0, S_{i}=360-S_{i}$, go to (3). 


\section{Some Illustrations of the Results for Long and Short Paths}

Recordings of VLF signals propagating over long VLF paths (> 15,000 $\mathrm{km}$ or so) often show diurnal variations in phase and amplitude which are indicative of at least some energy coming over the long great circle path [Crombie, 1958], particularly when the short path is completely or almost completely sunlit. This makes the variation of daylight or darkness over both the long and short paths especially interesting.

Figures 3 and 4 show typical sets of the computer output for sunrise and sunset calculations for the short and long path from GBR, Rugby, England, to Byrd Station, Antarctica, for April 15, 1962。 The upper set in each figure is taken for an ionospheric height of $90 \mathrm{~km}$, and a screen height of $27 \mathrm{~km}$, or $\chi=98^{\circ}$. The lower set in each figure is for ground level or $x=90^{\circ}$. These figures illustrate several interesting points. First is the essentially complementary nature of the two paths, i.e., one is becoming light when the other is becoming dark, and vice versa. Ant other is the extremely rapid transition, especially at ground level, around 1900 UT. This could be expected to cause a very rapid transition of the predominant component of the received signal from the long path to the short path. This effect occurs when the great circle joining the transmitter and receiver nearly coincides with the terminator. It is indicated by a solar and transmitter bearing difference close to $90^{\circ}$ or $270^{\circ}$ at sunrise or sunset. 
These effects are also illustrated in figures 5 and 6 . These show the output plot of percentage of path in darkness, distance along path of terminator location, the solar bearing angle, and the cosine of the solartransmitter bearing angle difference at sunrise and sunset. The rapid transition occurs when the bearing angle at which the path crosses the equator $\left(9.63^{\circ}\right)$ is nearly equal in magnitude to the solar declination $\left(9.64^{\circ}\right)$. The bearing at which the path crosses the equator is also equal to the complement of the maximum latitude ( $\left.80.37^{\circ}\right)$ on the great circle path. The bearing difference angle is interesting, not only because of its influence on the time interval during which the night-day. transition is made, but also because experimental data indicate that the angle at which the terminator crosses the path appears to influence the amount of mode conversion at the discontinuity in the waveguide height caused by the terminator [Crombie, 1964].

Figure 6 illustrates an interesting characteristic of some paths at certain times. Note that the path at ground level remains about $80 \%$ dark until 1850 UT. The percent of darkness then drops to $18 \%$ in about fifteen minutes. The percentage in darkness at the ionospheric height changes gradually from about 1430 UT. It begins to drop rapidly after 1700 and reaches zero at 1825 UT. It then rises from zero to $10 \%$ between 1950 and 2120 UT.

A corresponding phase retardation at about the proper time (1958 UT in this case) on a path showing such behavior may enable one to determine the critical value of $x$, at which the ionizing radiation first affects the D layer. 


\section{Acknowledgments}

The author is indebted to D. D. Crombie for his suggestions which added considerably to the usefulness of these programs. The work was sponsored by the Advanced Research Projects Agency, Washington, D. C., under Order No. 183.

\section{5. $\underline{\text { References }}$}

Brady, A. H., and D. D. Crombie (1964), Calculation of sunrise and sunset times at ionospheric heights along a great circle path, NBS Technical Note No. 209.

Crombie, D. D. (1958), Differences between the EW and WE propagation of VLF signals over long distances, J. Atmos. Terrest. Phys. 12, 110117 .

Crombie, D. D. (1964), Periodic fading of VLF signals received over long paths during sunrise and sunset, Radio Sci. J. Res. NBC 68D, No. I, $27-34$. 


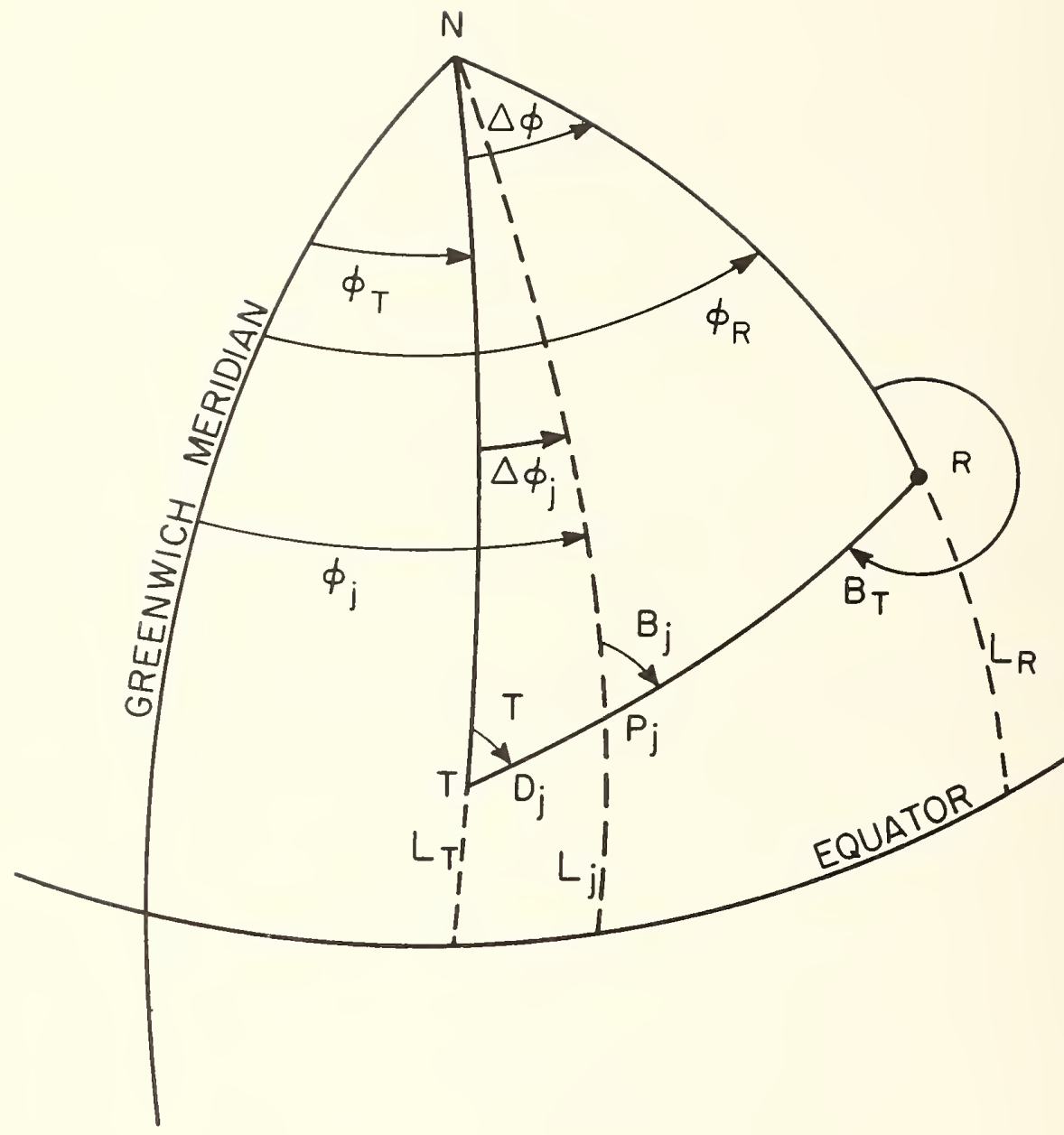

Figure I

Geometry of a Great Circle Path 


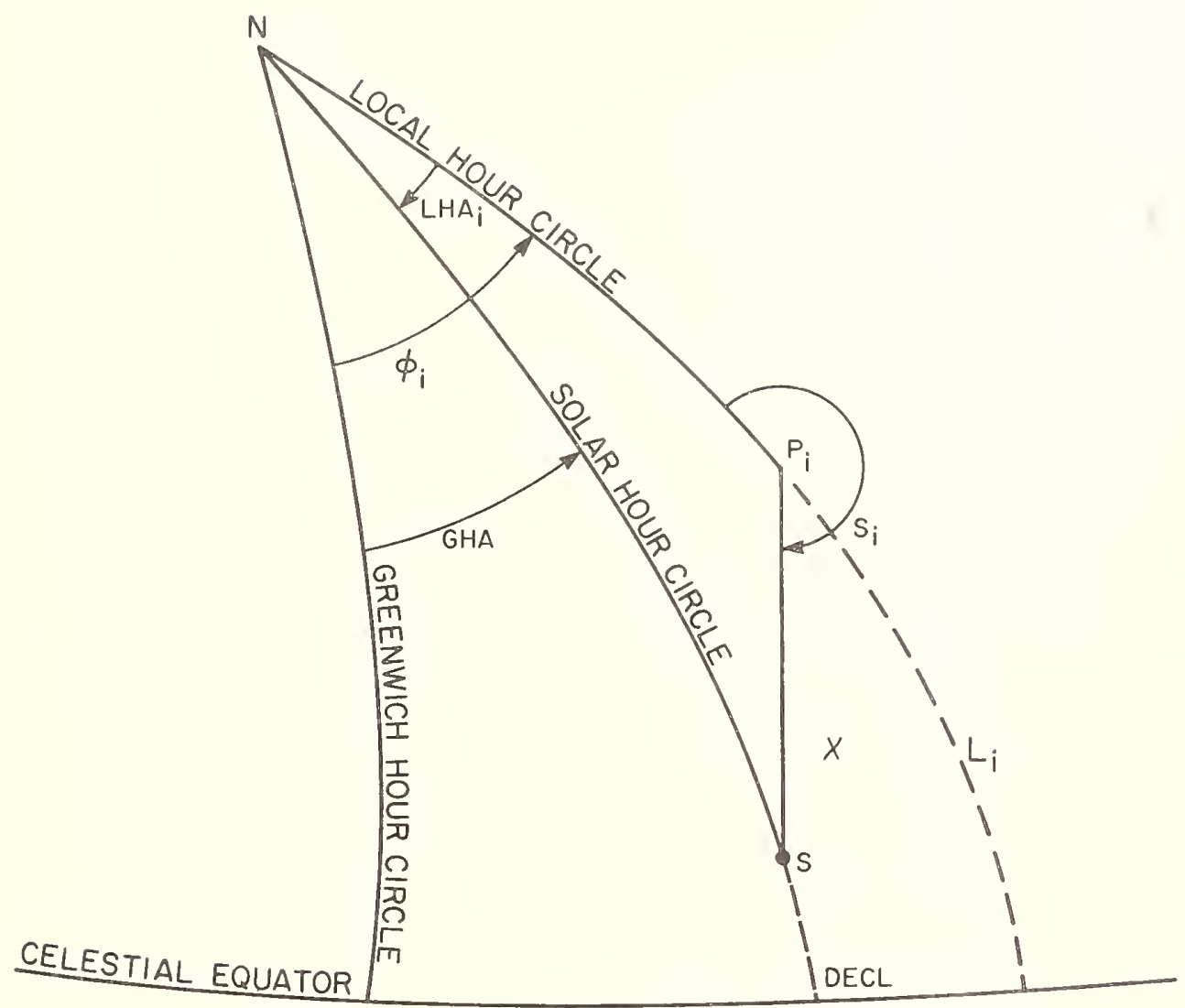

Figure 2

The relationship of the solar local hour angle, zenith angle, declination and bearing angle. 

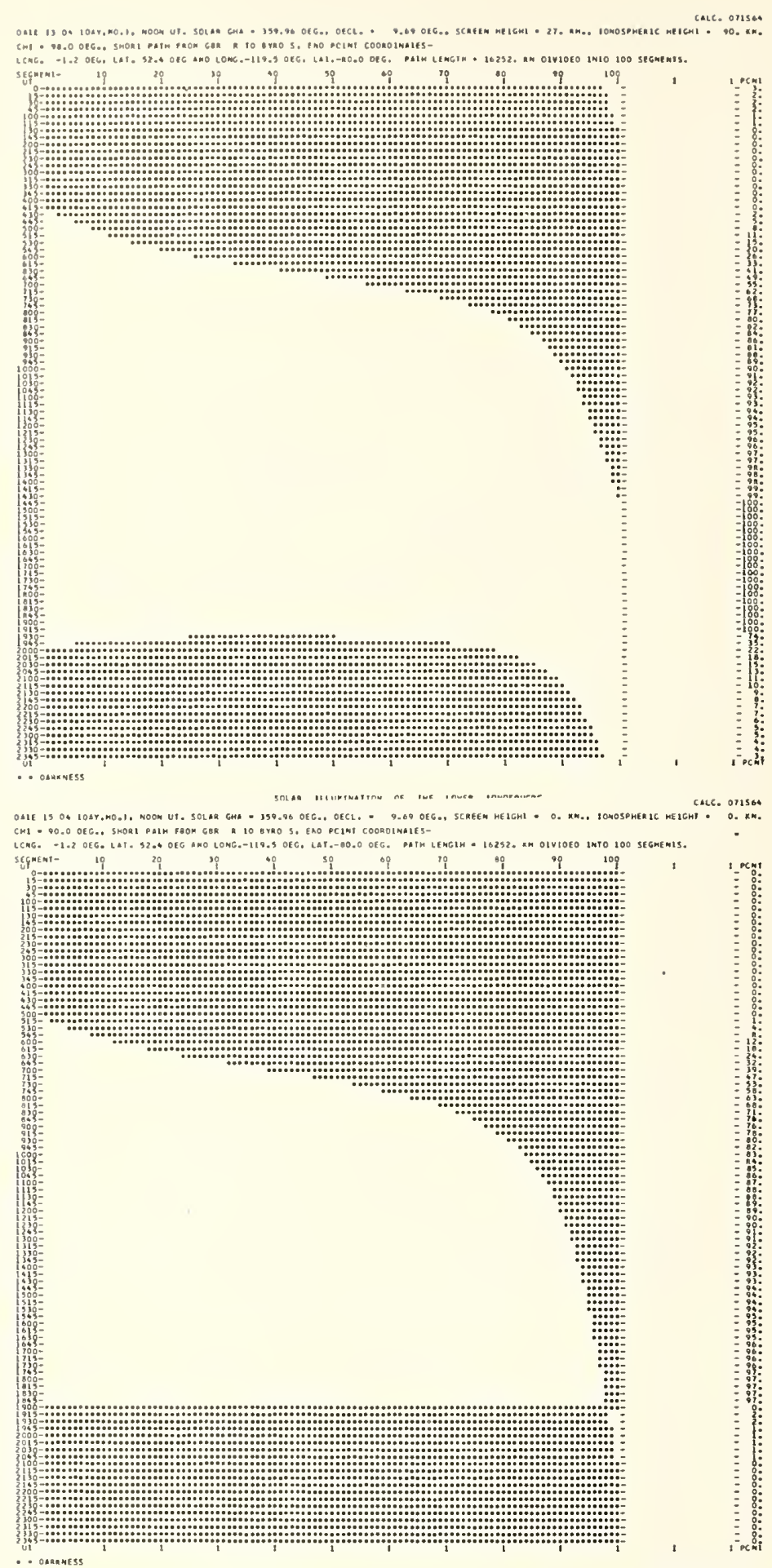

Figure 3. Light and Darkness Plot vs. UT from the Short Path from GBR, Rugby, England, to Byrd Station, Antarctica for 15 April (1962), for (a) ground or $x=90^{\circ}$, and 

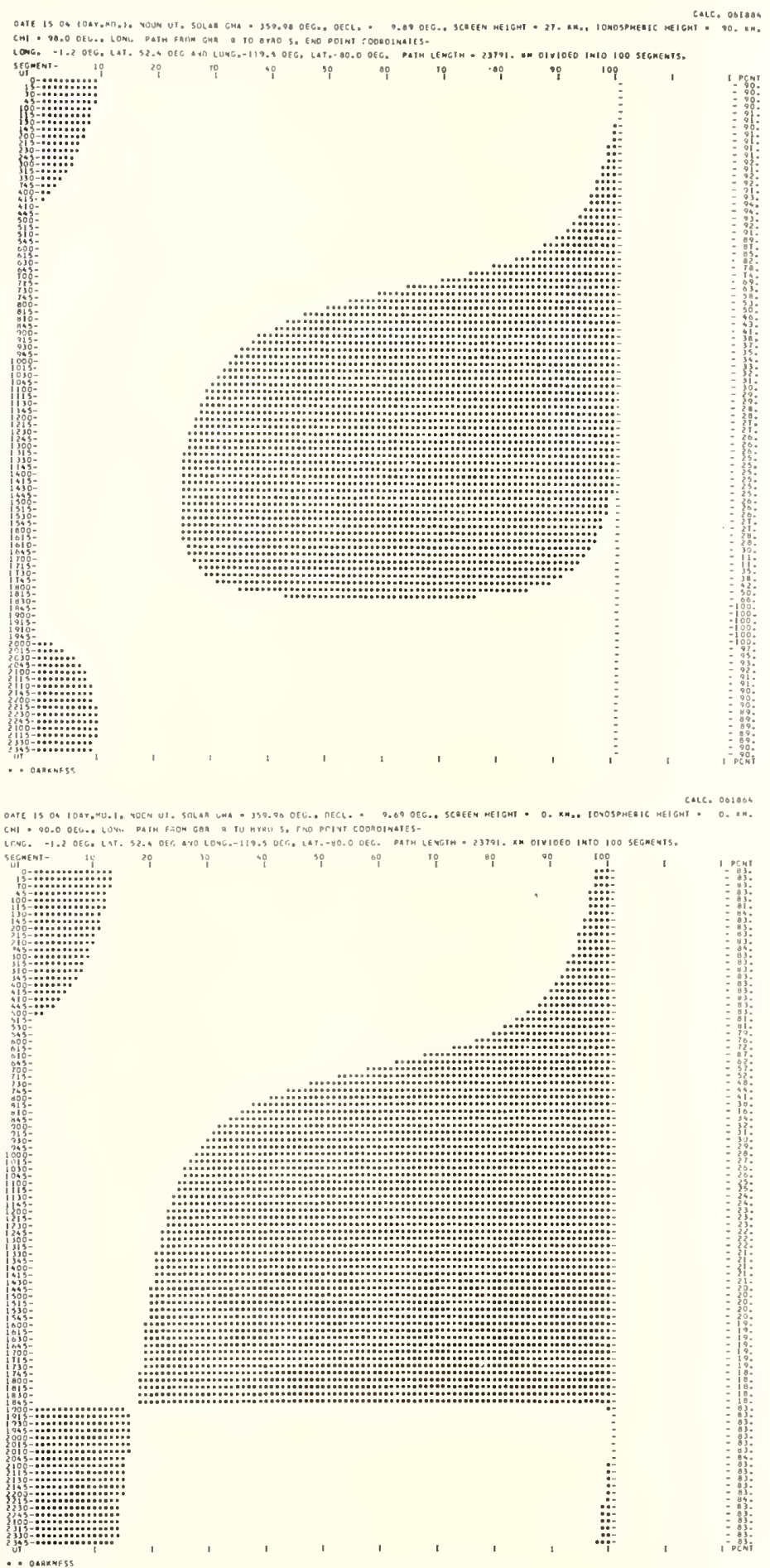

Figure 4. Light and Darkness Plots vs. UT for Long Path from GBR, Rugby, Engl Byrd Station, Antarctica for 15 April (1962), for (a) ground or $X$ (b) ionospheric reflection height $=90 \mathrm{~km}$, screen height $=27 \mathrm{~km}$, o 


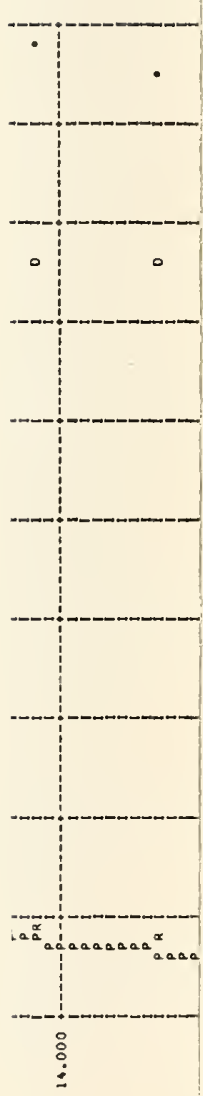

Figure 


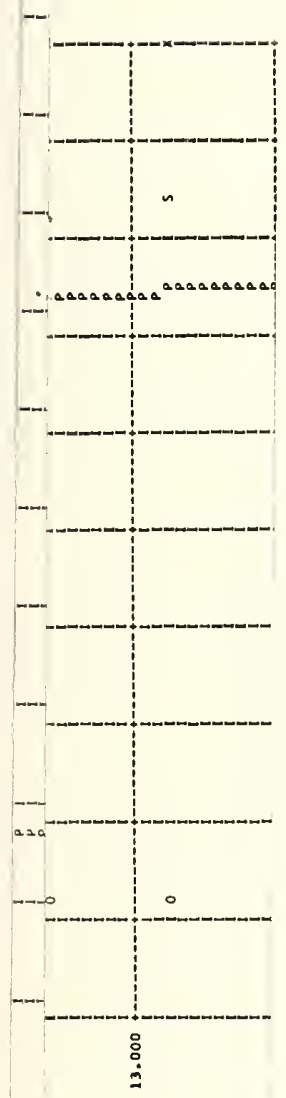



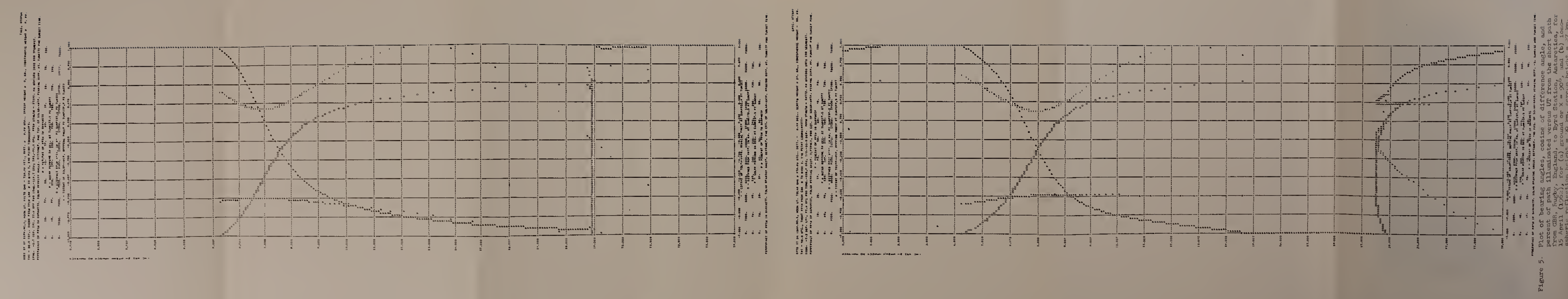

(19.8) 